\title{
Characterizing complex impedance in TES Detectors for SuperCDMS
}

Nishant Mishra ${ }^{a}$, Noah Kurinsky ${ }^{b}$

\section{Introduction}

Dark Matter composes $70 \%$ of all matter in the universe and traces out the large-structure of the universe. The SuperCDMS detector attempts to directly detect thermal relic dark matter, probing down to $300 \mathrm{MeV}$ through nucleus scattering and $500 \mathrm{keV}$ through electron scattering. To accomplish this we make use of Transition Edge Sensors (TES). TES-based detectors sit in the transition regime between acting as a superconductor or a normal conductor. As particles hit the detector, they heat it changing the impedance. To characterize this process we measure the impedance at multiple DC bias points (Figure 3) and multiple temperatures (Figures 5, 6).

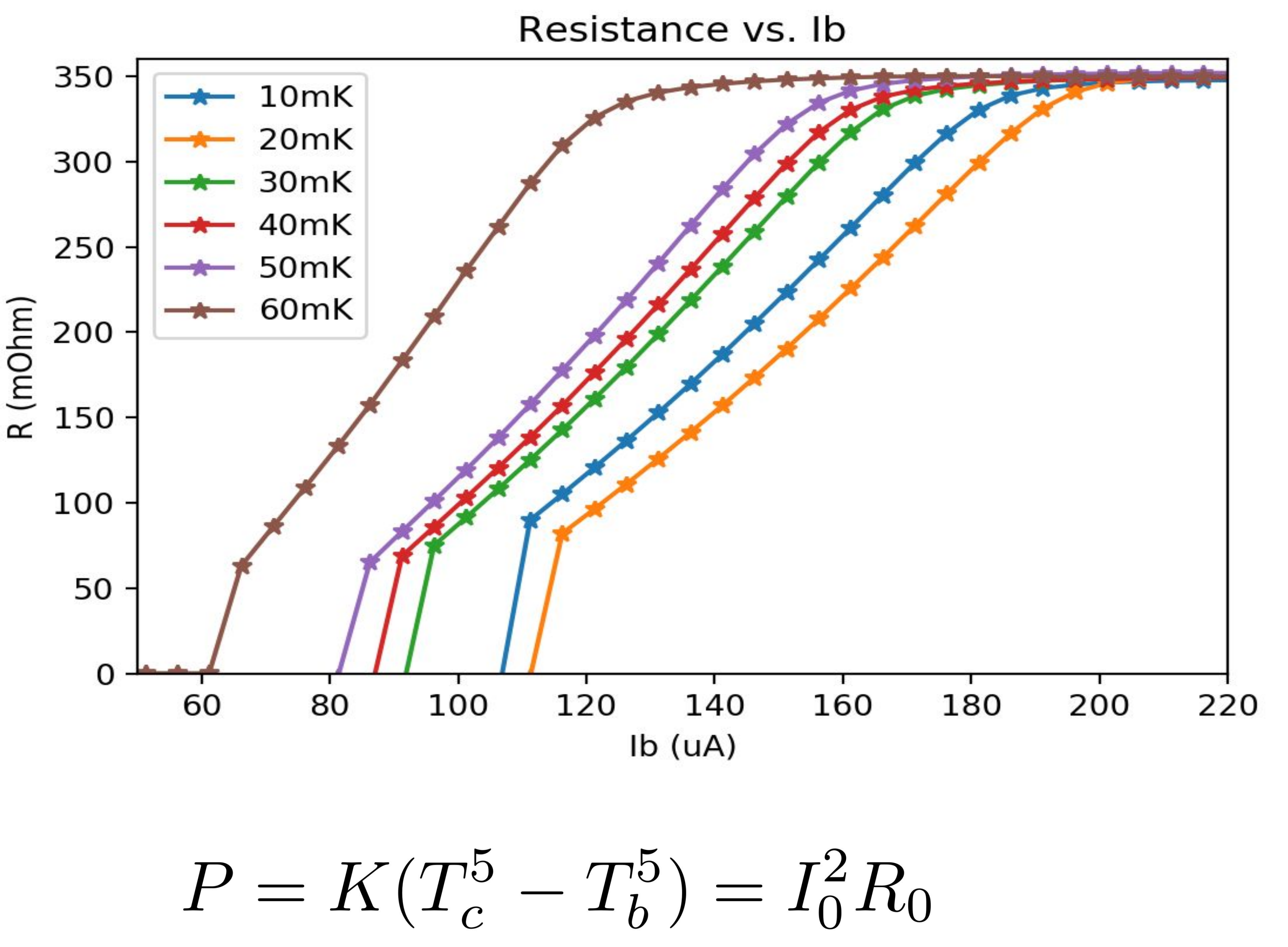

Figure 2: Showing effective resistance as a function of DC bias point at a multitude of detector temperatures. We measure the impedance at each of these points by sending an AC signal. The TES power equation is also given. It describes the lower power/current requirements at higher bath temperatures. $T_{c}$ is higher than all our test temperatures, so the system needs to draw less power to raise the temperature to match.

\section{Block Models}

The most basic model of the thermal circuit is a single heat capacity connected to a heat bath with one thermal

conductance. However, modern detectors show that there is a more complex response from detectors. When fitting complex impedance data we model the detector as two or three blocks. Each block is assumed to have a seperate heat capacitance and can describes and additional thermal body in the detector.
Figure 3 diagrams two-block model, which can be analagously imagined as coupled harmonic oscillators with different heat capacitances acting as springs with different spring constants. The primary purpose of our project is to characterize the complex impedance of our detector in terms of the three-pole models.
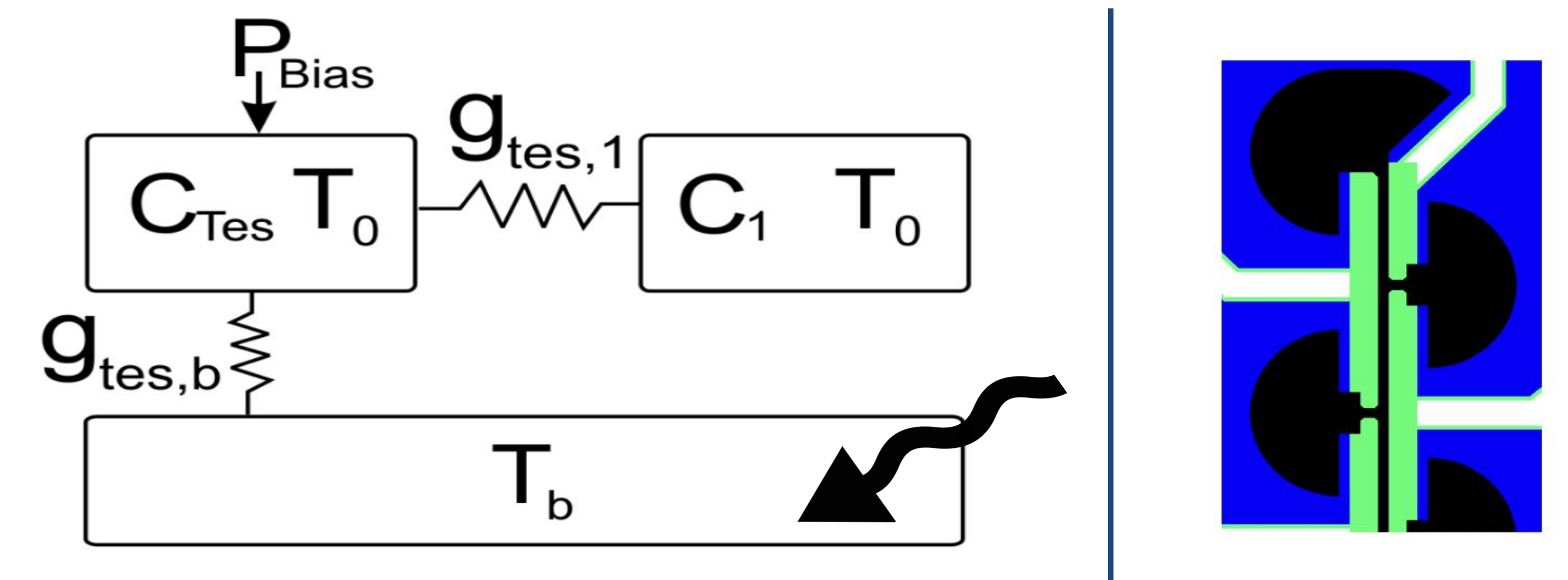

Diagram of the two block model (Maasilta, 2012), whose distinguishing feature is an additional body with a seperate heat capacity and thermal

Fitting along the transition

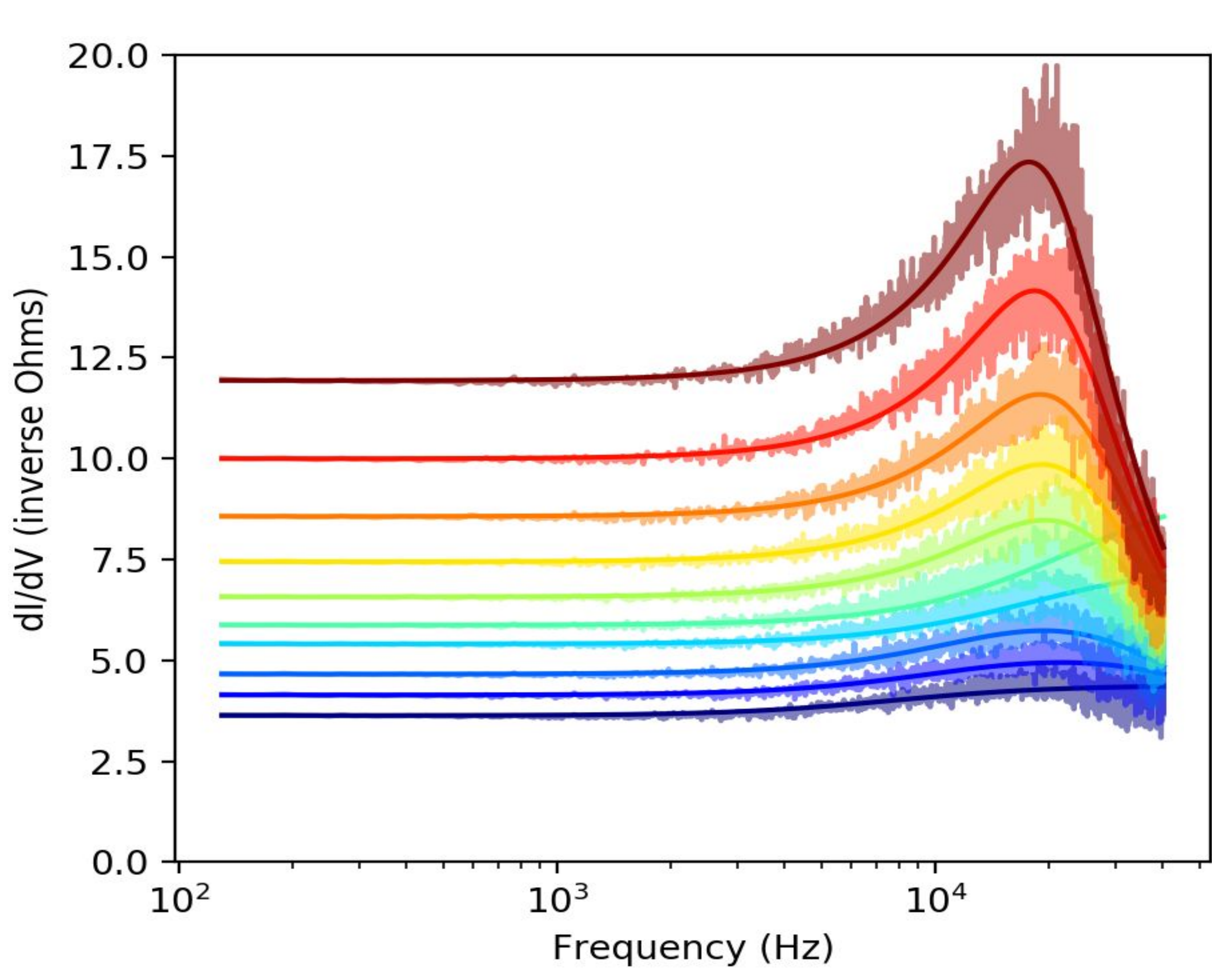

igure 3: We fit the 2 block model along each of the bias points. We find that higher bias power results in a higer admittance, and the increased prominence of the third pole is greater at lower bias points. We also note that the fit does not match well at high frequencies and

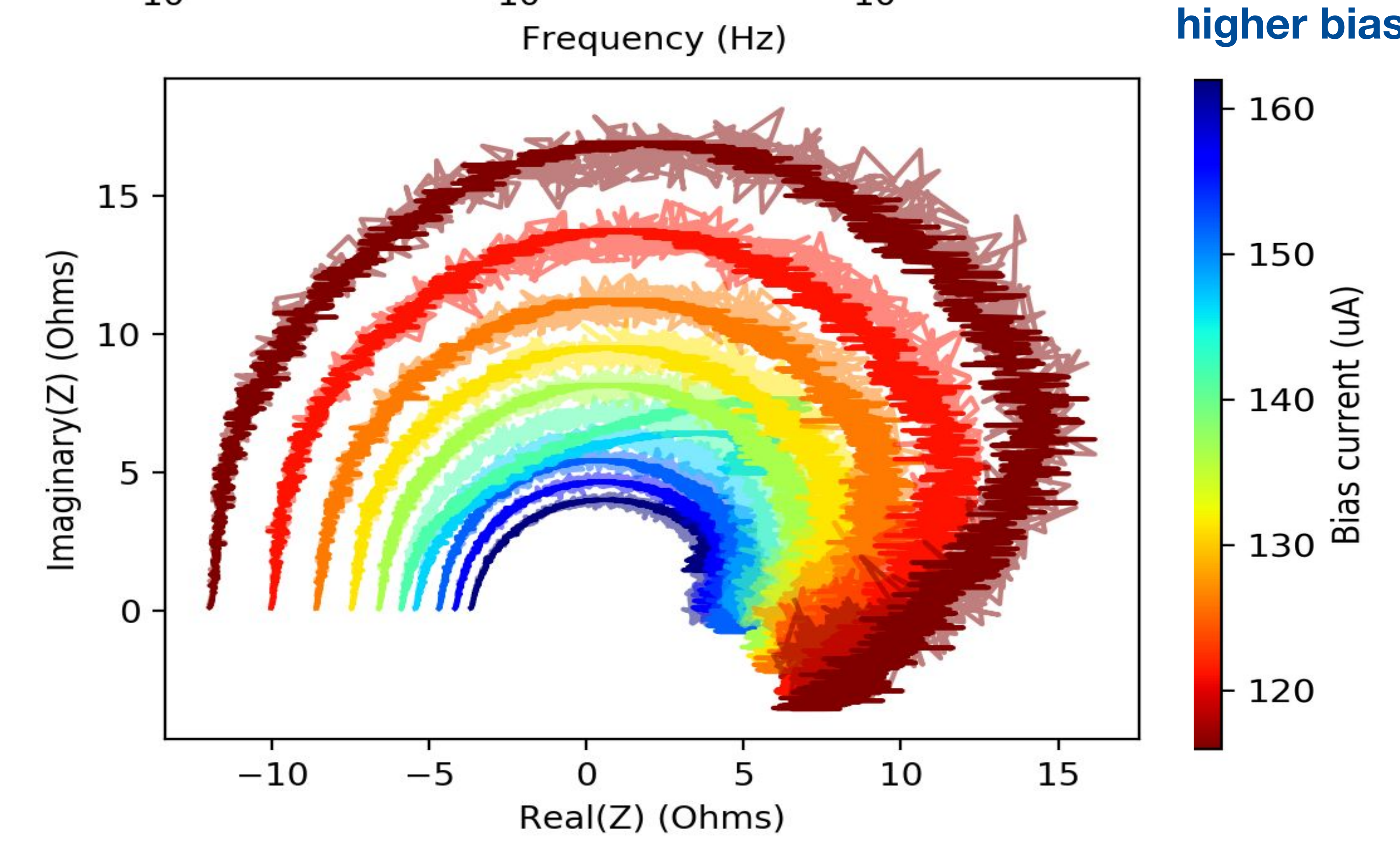

\section{Varying Temperature}

There is evidence that the two block model is effective for TES detectors at higher temperatures. Looking at previous detector data, the three block model can better recreate response of the circuit. Our reasoning is that . $g_{\text {tes, } 1}$ is the linearized thermal conductance and it proportional to the difference in temperature between the two blocks it connects. Similarly for $g_{\text {. }}$. So, if we raise the bath temperature it stands to reason that ratio between the conductances would fall. We check this reasoning with the newest detector by taking data at multiple temperatures and fit to find this ratio in Figure 4. In Figure 5, we describe the effective time constant as a function of temperature and bias point, which is consistent with expectations.

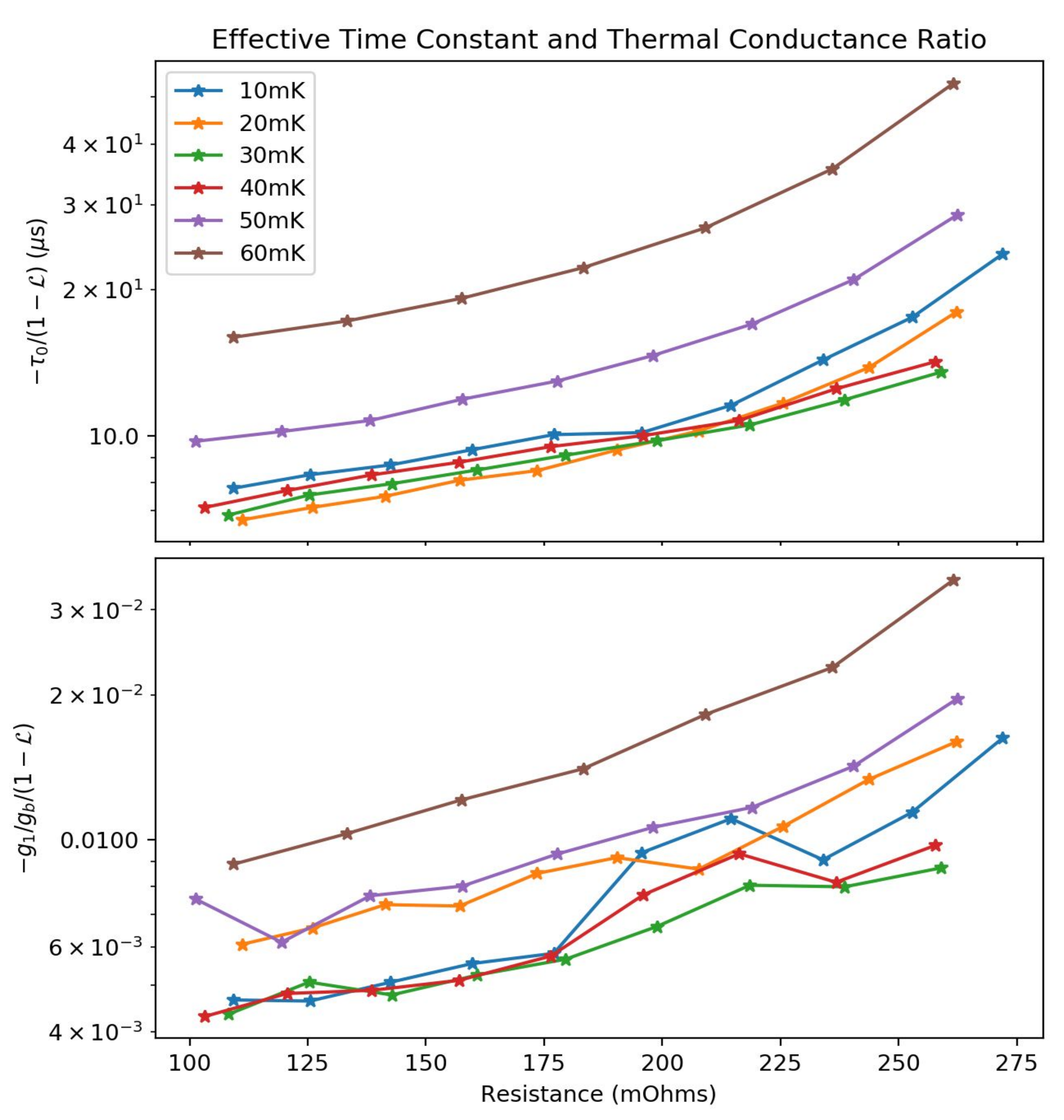

Conclusion and Acknowledgements

In the future, we will use these results to perform more detailed noise modelling on the detector. We like to thank Nick Mast,

Samuel Watkins, Zachary Williams, Ran Chen, Ziqing Hong, and many others in the SuperCDMS collaboration for their comments and assistance with this project. 